\title{
LA ACTUALIDAD CONSTITUCIONAL EN ESTADOS UNIDOS EN 1999
}

\author{
MARCOS CRIADO Y ANTONIO DE CABO \\ Departamento de Derecho Constitucional \\ Universidad Complutense
}

La presente revisión de la doctrina constitucional norteamericana se estructura en cuatro epígrafes. En el primero, se analiza el debate entorno al segundo volumen del libro We the people de B. Ackerman; en el segundo, se estudia la reconsideración del federalismo emprendida con base en ciertas decisiones jurisprudenciales; en el tercero, se vuelve sobre el problema de la financiación escolar (analizado desde el punto de vista jurisprudencial en revisiones anteriores ${ }^{\mathfrak{1}}$ ); y en el cuarto, por último, se realiza un somero repaso de la jurisprudencia constitucional norteamericana (leading cases).

Con carácter general, cabe señalar un cierto cambio en la orientación de las preocupaciones de la doctrina al que, acaso, no sea ajena la proximidad de la finalización del período presidencial de B. Clinton. En efecto, parece haber desaparecido la inquietud por el ejecutivo, tanto en su vertiente más política como puramente administrativa (agencias, etc.), trasladándose hacia el poder judicial. Pero no como hasta ahora, en lo relativo al problema de las relaciones entre legislativo y judicial, sino en lo que se refiere a las relaciones entre poder judicial y estructura constitucional, es decir, entre poder judicial y federalismo (volviendo, en parte, al problema inicial que hizo surgir este tipo de jurisdicciones federales). En este sentido, los dos primeros bloques de esta revisión se refieren a problemas de estructura

1. Marcos CRIADO y Antonio DE CABO, "La actualidad constitucional en Estados Unidos en 1997", Teoria y Realidad Constitucional, núm. 1; 1998, pp. 248-249; y Marcos CRIADO y Antonio DE CABO, "La actualidad constitucional en Estados Unidos en 1998", Teoría y Realidad Constitucional, núm. 3, 1999, pp. 305 . 
constitucional: el primero, a los cambios constitucionales (en último término, a las relaciones entre reforma y mutación constitucional en el peculiar contexto norteamericano); el segundo, a las relaciones entre estados y poder federal, y a su mediación por el poder judicial. Ya no se trata tanto del problema de la objeción contramayoritaria como de una nueva discusión del tradicional problema de la soberanía en los estados federales.

\section{BRUCE ACKERMAN Y LOS "MOMENTOS CONSTITUYENTES"}

Efectivamente, el debate doctrinal estadounidense durante el año 1999 viene en buena parte marcado por la publicación el año anterior del segundo volumen del libro del profesor Bruce Ackerman We the people ${ }^{2}$, donde completa la revisión de los momentos constituyentes de la Federación los Estados Unidos de Norteamérica, y adelanta una teoría del hecho constituyente o del cambio constitucional. Las respuestas doctrinales que ha suscitado esta revisión de la historia constitucional norteamericana serán puestas aquí de relieve desde dos puntos de vista distintos: uno, de carácter histórico, pone en duda que los períodos históricos elegidos por el autor sean verdaderamente constituyentes, aun según los caracteres mismos con los que el profesor Ackerman caracteriza el cambio constitucional, al tiempo que señala otros momentos de la historia norteamericana como momentos en que la Constitución habría sufrido cambios determinantes. El otro, más especulativo, analiza los problemas que la adopción de una "teoría constitucional", entendida como teoría "sobre la naturaleza de la Constitución de los Estados Unidos y sobre cómo debe ser aplicada por los jueces" ${ }^{3}$, plantea en un sistema de common law donde se espera que los jueces participen en la creación del Derecho del Estado resolviendo las controversias caso por caso, sin someterse a los principios generales e invariables que toda teoría comporta ${ }^{4}$.

Ya en 1984, Bruce Ackerman había diseñado su concepción dualista de la Constitución norteamericana, donde existirían dos procesos legislativos distintos, uno ordinario y otro en que la soberanía del pueblo se ejerce con todas sus consecuencias para dar sentido a las previsiones constitucionales 5 . Así, la Constitución norteamericana habría sido informalmente reformada por enmiendas no escritas, y no aprobadas según el proceso previsto en su artículo $\mathrm{V}$, reformas que habrían sido

2 Bruce AcKerman, We the People: Trasformations, Cambridge, Mass., Harvard University Press, 1998. El volumen anterior, We the People: Foundations, fue publicado por la misma editorial en 1991. p. 537.

3. Richard H. FAllon, How to choose a Constitutional Theory, 87 Californian Law Review, 1999,

4 El rechazo de una teoría para ser aplicada por los jueces en la resolución de las controversias en que se invoque la Constitución estadounidense es el núcleo del "pragmatismo metodológico" del juez Richard A. POSNER en su artículo Against Constitutional Theory, 73 New York Law Review, 1998, p. 1 y ss. La inutilidad de una teoría tal es defendida por Cass R. SunsTeIn, The Supreme Court, 1995 Term-Foreword: Leaving Things Undecided, 110 Harvard Law Review, 1996, pp. 14 a 21.

5. Bruce ACKERMAN, The Storrs Lectures: Discovering the Constitution, 93 Yale Law Journal, 1984, p. 1013. La idea de la "Constitución dual" viene también expuesta en We the People: Foundations, op.cit., pp. 3-33. 
legitimadas a través de una ratificación popular diferente ${ }^{6}$. Es más, las grandes reformas de la Constitución norteamericana se habrían llevado a cabo conforme a un proceso no constitucionalmente tasado, que permitiría que fuera la soberanía popular el verdadero motor de las reformas, así como la posibilidad de una auténtica interpretación judicial concebida como vista atrás, como labor de conservación de la norma superior determinada por el ejercicio de la soberanía popular, y no como activismo judicial para la adecuación del texto constitucional a una nueva realidad?. A este proceso de reforma que ha gobernado las transformaciones constitucionales independientemente del proceso de enmienda previsto en el artículo V, lo denomina higher lawmaking norms, y a la exposición de los dos momentos históricos en que ha sido utilizado, aparte del momento fundacional del Estado norteamericano, dedica el segundo volumen de We the People ${ }^{8}$. Estos dos momentos son:

- Mientras Lincoln, y después Johnson, habían presionado a los Estados del Sur para que ratificaran la decimotercera enmienda, Johnson no hizo lo mismo con la decimocuarta, y permitió que las elecciones de 1866 se convirtieran, según Bruce Ackerman, en "one of the great higher lawmaking events of American history" ${ }^{9}$. Los republicanos acudieron a las elecciones haciendo de la decimocuarta enmienda el núcleo de su programa y, según la teoría de Ackerman, consiguieron un mandato popular para reformar la Constitución. Cuanto menos, así lo interpretaron, porque cuando Johnson permitió el veto reconocido en el artículo $\mathrm{V}$ de la Constitución a diez Estados del Sur, el Congreso republicano respondió desmantelando los gobiernos rebeldes de su mismo partido, y exigiendo la ratificación de la enmienda a cambio del reconocimiento de los gobiernos nuevamente formados. Cuando Johnson se opuso al plan, le abrieron un proceso de impeachment, y al final acabó sometiéndose a la interpretación constitucional de la mayoría del Congreso, sentando, en opinión de Ackerman, el precedente del Switch in Time, es decir, de la permisividad del Tribunal Supremo conservador de 1937 para con las medidas del New Deal tras el triunfo electoral del año anterior.

6 Bruce AcKerman, We The People: Foundations, op.cit., pp. 44-50, 81-130; Id., The Storrs Lectures, op.cit., pp. 1057-1070; Id., We the People: Transformations, op.cit., p. 270: Id., Constitutional Politics/Constitutional Law, 99 Yale Law Journal, 1989, p. 453.

7. Bruce ACKERMAN, We the People: Foundations, op.cit., pp. 131-162.

8 El proceso de higher lawmaking cuenta con cinco fases: "Signalling", "proposing", "triggering", "ratifying" $\mathrm{y}$ "consolidating" (We the People: Transformations, op.cit., pp. 32-68), fases que según el autor no pueden acomodarse a las normas prescriptivas previstas para las enmiendas ni está relacionado con la legitimidad del cambio constitucional prevista por los Padres fundadores, porque es el proceso con el que la misma fundación del Estado se produjo. Este mismo esquema se aplica al momento de la Reconstruction policy, a mediados del siglo pasado, durante las presidencias de Lincoln y Johnson (pp. 99-252), lo que le permite diferenciar los momentos políticos que provocan el proceso: (a) Paso de un liderazgo presidencial a un liderazgo del Congreso; (b) Los conflictos entre ambos poderes del Estado se plantean como peligrosos para el principio de separación de poderes y (c) a quién pertenezca el mandato popular se resuelve mediante elecciones nacionales, que dan la razón a una de las partes enfrentadas (pp. 236-251). Este mismo esquema se aplica a las vicisitudes de la instauración de las políticas del New Deal durante la presidencia de F. D. Rooselvelt.

9. Bruce AcKerman, We the People: Trasformations, op.cit., p. 103. 
- El mismo esquema se reproduce durante la época del New Deal, cuando, tras el bloqueo del Congreso y del Tribunal Supremo a las reformas de los New Dealers, éstos ganaron tanto las elecciones al Congreso como las presidenciales de 1936, obteniendo el respaldo popular a su interpretación constitucional ${ }^{10}$.

En consecuencia, el bigher lawmaking es un proceso histórico de legitimación del cambio constitucional que consiste en (a) una situación de impasse constitucional, (b) un enfrentamiento institucional que pone en juego la separación de poderes, (c) la concesión de un "mandato" electoral, y (d) la puesta en marcha del Switch in Time. Este proceso nada tiene que ver con una teoría legal de la legitimidad de las reformas, sino que únicamente pretende poner de manifiesto una situación "constitutiva" en la que se ven involucrados en el proceso de reforma constitucional movimientos sociales, votantes, la clase política en general, y los representantes y el Presidente en particular. El problema es que Ackerman afirma, más que demuestra, la identificación entre las opiniones de las elites reformistas y las del movimiento popular. Para Ackerman, en los dos casos contemplados la voluntad popular era la que las elites dijeron que era, con lo que el centro de la legitimación del cambio que Ackerman entiende descubrir, es decir, la deliberación y participación popular en la gestación de la reforma, desapare$\mathrm{ce}^{11}$. La crítica se refuerza por el hecho de que Ackerman no analiza si las aspiraciones populares de reforma fueron después adecuadamente traducidas por la opción política vencedora, o si se transformaron y cómo a lo largo del proceso, o si existió algún tipo de transacción entre movimientos sociales, partidos políticos, representantes y Presidente.

En este sentido, hay autores que, desde un punto de vista estrictamente histórico, explican la legitimación de las reformas de la época de la Reconstruction no por su unión con los deseos del pueblo, sino en el contexto político y moral de los Estados Unidos de mediados del $\mathrm{XTX}^{12}$. Otros autores sugieren que la voluntad de los votantes blancos que respaldaron la decimocuarta enmienda bien podía ser la de castigar al Sur, y no, como pretende Ackerman, la de llegar a una concepción no racial de la ciudadanía ${ }^{13}$.

10. Una interpretación distinta del resultado de las elecciones de 1936 puede encontrarse en William E. Leuchtenburg, When the People Spoke, What Did They Say?: The Election of 1936 and the Ackerman Thesis, 108 Yale Law Journal, 1999, pp. 2.077 y ss. Sobre la distinta significación que dan los historiadores a los hechos y textos históricos manejados por Bruce Ackerman, cfr. en el mismo número de la revista Jack N. RAKove, The Super-Legality of the Constitution, or, a Federalist Critique of Bruce Ackerman's Neo-Federalism, op.cit., pp. 1937-1946 y Bruce ACKERMAN, Revolution on a Human Scale, nota 22.

11. Cfr. William E. Forbath, Constitutional Change and the Politics of History, 108 Yale Law Journal, 1999, pp. 1923 y 1924.

12. Cfr. Rogers SMITH, Legitimating Reconstruction: The Limits of Legalism, 108 Yale Law Journal, 1999, p. 2043 y Eric FoNER, The Strange Career of The Reconstruction Amendments, ibidem, pp. 2.006 y 2.007 .

13. Cfr. Walter DeAN Burnham, Constitutional Moments and Punctuated Equilibria: A Political Scients Confronts Bruce Ackerma's We the People, Ibidem, pp. 2267-2269 y Michael LES BENEDICT, Constitutional History and Constitutional Theory: Reflections on Ackerman, Reconstruction, and the Transformation of the American Constitution, Ibidem, p. 2.253. 
Ackerman tampoco tiene en cuenta importantes cambios constitucionales que no siguieron ni el proceso contenido en el artículo V de la Constitución, ni el proceso de legitimación del bigher lawmaking. Foner y Burnham hacen referencia a la reacción de finales del siglo XIX y principios del XX contra el aperturismo de la época de la Reconstruction (el llamado system of 1896) ${ }^{14}$ y que legitimó las medidas segregacionistas y de separación racial. Para Burnham, este fin de siglo cavernario vino en gran medida impuesto por el fraude y la coacción ${ }^{15}$, y en todo caso estuvo presidido por el activismo judicial del Tribunal Fuller ${ }^{16}$. Este tipo de activismo judicial, muy frecuente en la historia de Estados Unidos, no se adapta al esquema diseñado por Ackerman, que, por ello mismo, tampoco tendría en cuenta el activismo judicial, más cercano a nuestro tiempo, del Tribunal Warren.

Los "momentos" constitucionales señalados por Ackerman se definen como ocasiones de redefinición de los derechos de la ciudadanía. Es claro que la incorporación de toda la población negra a la condición de ciudadano, y por tanto, al disfrute de los derechos civiles con la aprobación de los Black Codes, colma la definición propuesta (al igual que la reacción posterior de segregación y apartheid), sin embargo, algunos autores cuestionan que pueda hablarse de una semejante redefinición de los derechos fundamentales en relación con el New Deall. En primer lugar, porque la retórica del New Deal, que fue acuñada por el ala izquierda de la coalición reformista, incluía el derecho a un trabajo decente y a un nivel de vida adecuado, un derecho a la previsión social y a la independencia económica que permitiera una auténtica democracia; estos derechos de los que hablaba Roosevelt ẹn su cámpaña de $1936^{18}$, quedaron reducidos a un genérico "paramount right to work" en su second Bill of Rights ${ }^{19}$. Este ejemplo bastaría ya para encontrar una falla entre el man-

14. La ruptura interna que, para el partido republicano, había supuesto el enfrentamiento de los representantes nacionales con el partido republicano sureño y sus gobiernos estatales durante la época de la Reconstruction, fue superada a través del populismo de Jim Crow, creando, durante estos años, un partido enormemente unido que hizo de la violenta reacción sureña contra el modo de vida impuesto, también violentamente, por el Norte, su bandera y su gran gancho electoral, hasta el punto de crear un sistema de partido único durante los años del "solid South". Cfr. William E. ForBATH, op.cit., p. 1.924 .

15. Walter DeAn BuRnham, op. cit, p. 2.265. Folder, en cambio, cree que existió un cierto tipo de legitimación popular (Eric FONER, op.cit., pp. 2.007-2.009).

16. Walter DEAN BURNHAM, op.cit., pp. 2.270 y 2.271

17. Cfr. William E. Forbath, op.cit., pp. 1.925-1.930.

18. Ibidem, p. 1.927.

19. En contra, Cass R. SunsteIn afirma que el second Bill of Rights sirvió precisamente para constitucionalizar el derecho a una asistencia social mínima (The Partial Constitution, Cambridge, Mass., Harvard University Press, 1993, pp. 138 y 139). Sin embargo, no debe olvidarse que el American Law Institute, a través del comité encargado por Roosevelt de elaborar un "Statement of Essential Human Rights" con ocasión de los trabajos preparatorios del Full Employment Bill declaró que "the place of social and economic rights in any modern declaration of rights of man had already been decided", $y$ que su apuesta era por una constitucionalización de derechos positivos, frente a las posturas que veían en los derechos sólo un componente negativo, de impedir la acción del Estado o del resto de los ciudadanos. Igualmente requerían una acción positiva del Estado para la instauración de un completo sistema de seguridad social, afirmando que "a system of social insurance is child's play in comparison with the system that gives effect to due process of law", John R. Ellingston, The Right To Work in Full Employment Act of 1945: Hearings Before a Subcomm. Of the Sen. Comm. On Baking E Currency on S. 380, 79th Cong. 1248-49 (1945), citado por William E. ForBaTH, op.cit., p. 1927. 
dato otorgado por el pueblo de acuerdo con el programa expuesto, y las concretas políticas (esto es el "momento" de cambio constitucional individualizado por Ackerman) desarrolladas por Roosevelt. La acción de los Dixiecrats (miembros sureños del partido demócrata) aliados con los republicanos del Norte para conseguir una mayor descentralización de la administración, así como la exclusión de las principales leyes sociales de amplias categorías de trabajadores del Sur, frente a las propuestas de cobertura universal y estándares nacionales para los programas de protección social, hicieron que estas últimas quedaran bloqueadas en el Congreso; el fraude y la intimidación que impidieron el voto en el Sur de los negros y los blancos pobres, es decir los sectores que podían quitar el poder en sus feudos a los Dixiecrats, dejaron la revolución constitucional del New Deal incompleta ${ }^{20}$. Por tanto, queda claro que el New Deal significó un considerable aumento de los poderes administrativos del gobierno nacional, aumento permitido por el Tribunal Supre$\mathrm{mo}^{21}$, pero la teoría de Bruce Ackerman no puede mostrar cómo se transformaron los derechos de los ciudadanos, por lo que la época del New Deal no podría ser identificada con un "momento" constitucional según su propia teoría ${ }^{22}$.

En último término, la teoría de Bruce Ackerman, podría entenderse como una teoría justificativa y una guía para futuras reformas constitucionales. Ahora bien, una teoría constitucional es también una guía para la aplicación judicial de la Constitución, y en este caso, para la aplicación de las reformas que en la estructura del gobierno federal introdujo el New Deal. Justamente por esta razón, las teorías de Ackerman han resucitado la reflexión sobre el valor de las teorías constitucionales ${ }^{23}$, sobre cómo una teoría constitucional puede juzgarse conforme a criterios substantivos de valoración, criterios que son los mismos que la labor de los jueces debería asegurar ${ }^{24}$. Estos criterios substantivos permitirían superar, al menos en cuanto al juicio que merezca una teoría en cuestión, la tradicional división de las teorías constitucionales norteamericanas entre positivistas (Text-Based Theories) ${ }^{25}$ y empiristas ${ }^{26}$

20. Cfr. Patricia Sullivan, Days of Hope: Race and Democracy in the New Deal Era, Chapel Hill, University of North Carolina Press, 1996.

21. Bruce ACKerman, We the People: Transformations, op.cit., pp. 279-382. Como resultado de esta enmienda implícita también habría cambiado la protección de la propiedad y de otros derechos económicos.

22. William E. ForBath, op. cit, p. 1930. Según ACKERMAn, la Full Employment Act debe ser considerada una parte de la Constitución del New Deal que We the People ha ratificado. Sin embargo, el autor no explica en ningún momento cómo las disposiciones de un texto de carácter programático como la Full Employment Act pueden obligar a nivel constitucional las políticas concretas de hoy en día.

23. Cfr. Richard H. Falion, Jr., How to Choose a Constitutional Theory, 87 California Law Review, 1999, pp. 535-579; David A. STrauss, What is Constitutional Theory?, ibidem, pp. 581-592; Michael C. DORF, Create your Own Constitutional Theory, ibilem, pp. 593-612.

24. Richard'H. Fallon, op.cit., p. 538.

25. Algunos representantes son Robert H. BORK, The Temping of America: The Political seduction of the Law, New York, Simon \& Schuster, 1991, y Antonin SCALIA, A Matter of Interpretation, Princeton, NJ, Princeton University Press, 1997.

26. Entre los que estarian el propio Bruce Ackerman, para quien la Constitución no es solamente el texto constitucional, sino también las enmiendas no escritas; Ronald Dworkin, Freedom's Law. The Moral Reading of the American Constitution, Cambridge, Mass., Harvard University Press, 1996; ID, Law's Empire, Cambridge, Mass., Harvard University Press, 1986; David A. STRAuss, op. cit. 
(Practice Based Theories). Según el profesor Richard H. Fallon Jr., las teorías constitucionales deben ser juzgadas según su capacidad para (a) mantener el principio de legalidad; (b) asegurar la justa competición democrática y (c) proteger un conjunto aceptable, moral y políticamente, de derechos fundamentales ${ }^{27}$.

La admisión de enmiendas constitucionales fuera del procedimiento tasado en el artículo V parece contraria en esencia al principio de legalidad ${ }^{28}$; $\sin _{\text {embargo, el }}$ propio Ackerman considera que sólo su teoría permite superar una interpretación constitucional de los jueces basada en sus propias opiniones políticas ${ }^{29}$, así como explicar la supervivencia del principio de legalidad en un ordenamiento constitucional donde la acción judicial es inexplicable por su sola referencia al texto constitucional $^{30}$. Según Ackerman, la posibilidad de que una ciudadanía movilizada políticamente pueda establecer normas constitucionales -que, parece ser, es el núcleo de su teoría-, ofrece, en primer lugar, más y mejores garantías de reconocimiento y defensa de un conjunto de derechos fundamentales de lo que lo haría la legislación ordinaria $^{31}$, y en segundo lugar, permite una adecuada estructuración del proceso democrático, ligándolo al principio de soberanía popular ${ }^{32}$.

\section{FEDERALISMO Y JUDICLAL REVIEW}

El término federalismo es generalmente utilizado por los constitucionalistas norteamericanos para designar a la doctrina que defiende los derechos de los Estados, es decir, que protege los derechos de los Estados frente a la acción federal. El tema que aquí se discute es si y cómo la soberanía de los Estados limita los poderes del Gobierno nacional, al hilo de la revitalización que en los últimos años parece haberse producido, tanto en la doctrina ${ }^{33}$ como en la jurisprudencia ${ }^{34}$, de la necesidad de

27. Richard H. Fallon, Jr., op.cit., pp. 549-557.

28. Robert H. BORK, op.cit., p. 215, donde describe la teoría de Ackerman como "an invitation for coup by judiciary".

29. Bruce ACKERMAN, Storrs Lectures, pp. 1.070-1.072.

30. Bruce Ackerman, We the people: Transformations, op. cit, p. 30. Aunque no existan normas claras que presidan la formación de las enmiendas no escritas, éstas se pueden identificar a través de los principios del ordenamiento y de los precedentes, ya que uthere is more to law than rules".

31. Bruce AcKerMan, We the people: Foundations, op.cit., p. 318. Cfr. también Suzanna ShERRY, The Gost of Liberalism Past, 105 Harvard Law Review, 1992, pp. 933 y 934, donde argumenta que la teoría constitucional de Ackerman permitiría proteger contra los ataques conservadores el liberalismo del New Deal convirtiéndolo en un momento de fundación constitucional.

32. Bruce ACKERMAN, We the people: Transformations, op. cit, p. 409.

33. Cfr. Vicki C. JACKSON, Federalism and the Uses and Limits of Law: Printz and Principle, 111 Harvard Law Review, 1998, p. 2.181, donde afirma que los tribunales should review the adequacy of congressional process to justify assertions of federal power"; Stephen Gardbaum, Retbinking Constitutional Federalism, 74 Texas Law Review, 1996, p. 795: "Courts should police Congress deliberative processes and its reasons for regulating to ensure that Congress seriously considered federalism concerns and adequately justified federal government action".

34. Cfr. Prinz v. United States, 521 U.S. 899 (1997) en que se declara la inconstitucionalidad de la Brandy Handgun Violence Protection Act y establece el llamado anticomandeering principle en las relaciones entre gobierno federal y Estados federados. Lo que el Juez Stevens (disidente en 
un control judicial de la adecuación al principio federal, entendido como reparto y equilibrio de poderes y competencias entre el gobierno federal y los Estados miembros, de la acción del legislativo federal. Este amplio problema ha sido, básicamente, abordado desde dos perspectivas, la primera se refiere a las relaciones entre jurisdicción estatal y jurisdicción federal ${ }^{35}$. La segunda, a la capacidad legislativa del Congreso en materia de Derechos Fundamentales (Enmienda 14, sección 5).

En la primea línea de interpretación del principio federal, Robert D. Scaphiro defiende un fuerte papel de los tribunales federales en la interpretación de las constituciones de los Estados ${ }^{36}$. Una razón a favor de este tipo de interpretación se halla en la politización de los jueces estatales. Mientras los jueces federales son funcionarios federales, nombrados de por vida por el Presidente de los Estados Unidos, los jueces estatales forman parte del sistema político del Estado y, por lo general, están sujetos a alguna forma de control electora ${ }^{37}$. Por otra parte, una completa eliminación de una judicial review federal de los actos administrativos de las agencias estatales impediría la unificación de los estándares de control judicial de la acción administrativa, con lo que podrían crearse niveles distintos (estatal y federal, distintos niveles estatales) de exigencia judicial de acomodación a la Ley y a la Constitución, y por tanto, niveles distintos de permisividad para con la discrecionalidad administrativa. Ello provoca problemas de seguridad jurídica y puede falsear la libre competencia en el comercio entre Estados $^{38}$.

La convivencia de dos órdenes constitucionales distintos en el Estado federal estadounidense, central y estatal, y la ausencia de una centralización para resolver las cuestiones constitucionales, provoca problemas de jurisdicción, y por tanto de interpretación constitucional, a la hora de asignar los casos en que se dilucidan cuestiones constitucionales, a uno u otro orden jurisdiccional. Al constitucionalizarse los mismos derechos en los Estados que en el Estado federal, se pueden presentar demandas ante ambas instancias, de forma que se producen interferencias entre los dos circuitos. No debe perderse de vista que lo que está en juego es simplemente la resolución del caso. La interpretación autoritativa del Derecho estatal compete a

esta promoción del principio federal) ha denominado athe Court's aggressive sovereign immunity jurisprudence" (Florida Prepaid, 119 S. Ct. at 2219 (Stevens, J., dissenting)).

35. Richard H. Falion, Daniel J. MELtzer \& David L. Shapiro, Hart and Wechsler's The Federal Courts and the Federal System (1999).

36. Robert D. SCAPHIRO, Polyphonia Federalism: State Constitutions in the Federal Courts, 87 California Law Review, 1999, pp. 1.409 y ss.

37. Sobre las diferencias institucionales entre jueces federales y jueces estatales cfr. C. HAZARD, Jr., Reflections on the Substance of Finality, 70 Cornell Law Review, 1985, p. 647. Es en el Estado de Tejas donde esta politización de las campañas para la elección de los jueces se ha sentido con más preocupación. Cfr. el comentario de Thomas R. Phildips en el vol. 61 de Law and Contemp. Probs, 1998, pp. 127 y ss, donde aboga por una reforma del sistema de elecciones judiciales en Tejas, porque los jueces que aceptan contribuciones económicas para su campaña, no pueden ser imparciales. Cfr. también Kate Thomas, Are Justices in Texas Getting Bought, National Law Review, Mar. 16, 1998 , a 8 , donde hace un report de las grandes contribuciones de los partidos a las campañas de los jueces.

38. Sobre el tema de la judicial review federal sobre la acción de las agencias estatales, cfr. Ann WoOlHANDDler y Michael G. Collins, Judicial Federalism and the Administrative States, 87 California Law Review, 1999, pp. 615 y ss. 
los órganos de la jurisdicción estatal ${ }^{39} \mathrm{y}$, en este punto, toda la estructura federal de jurisdicción, incluido el Tribunal Supremo, actúa como agentes de la jurisdicción estatal. ¿A qué orden jurisdiccional se confía la resolución de los casos en que se invoca la violación de una norma contenida tanto en la Constitución federal cuanto en la constitución del Estado por un acto o disposición de ese mismo Estado? La ausencia de una clara delimitación legal de jurisdicciones y competencias, así como la disparidad (e incluso la contradicción) de las soluciones interpretativas propuestas por el Tribunal Supremo, que actúan como precedentes, hacen del propuesto, un tema harto complicado, falto de una solución unívoca.

En general, la Ley atribuye a los tribunales federales la capacidad de tomar en consideración los recursos basados en el derecho estatal como jurisdicción diversificada ${ }^{40}$ (Rests on diversity) o suplementaria ${ }^{41}$ (supplemental). En un primer momento ${ }^{42}$, el Tribunal Supremo dispuso que cuando se trate de cuestiones constitucionales que incumban a la Constitución federal y a una estatal, es preferible que sean conocidas por la jurisdicción federal, que resolverá la cuestión conforme a la constitución estatal, intentando evitar, como canon interpretativo, la cuestión constitucional federal (federal constitutional claims avoidance).

Tal doctrina es contradicha por otras sentencias del Tribunal Supremo, tendentes a otorgar a la jurisdicción estatal los casos que claramente incumban cuestiones de derecho estatal (certain State-Law issues):

- Siguiendo el principio de evitar pronunciarse sobre cuestiones constitucionales federales superfluas, Railroad Commissión of Texas v. Pullman ${ }^{43}$ impone que la jurisdicción federal se abstenga de conocer de los casos atinentes al Derecho del estado, para que sea la jurisdicción estatal quien interprete su propia normativa. Así se evitarían innecesarios pronunciamientos constitucionales e interferencias con las concretas políticas desarrolladas en un determinado Estado ${ }^{44}$.

- La certificación (certification) es un proceso alternativo a la abstención. Según este proceso, el juez federal puede solicitar del juez estatal que se pronuncie sobre si se ha producido o no una violación de la constitución del Estado mientras el proceso se sustancia en el circuito federal. El problema es que (aparte de que no lo admiten todos los Estados) algunos de ellos sólo admiten certificaciones provenientes de los tribunales federales de apelación, y no de los tribunales de distrito. Igualmente, si bien es una solución expeditiva para los casos en que sólo el juez federal está conociendo de la controversia ${ }^{45}$, no lo es para los casos en que el litigio se substancia en ambas jurisdicciones simultáneamente.

39. Interpretación que debe ser acatada y aplicada por los tribunales federales. Cfr. Erie Railroad Co v. Tompkins, 304 U.S. 64 (1938).

40. 28 U.SC., parágrafo 1.332 (1994).

41. Id., parágrafo 1.367.

42. Siler v. Louisville E Nashville Railroad Co., 213 U.S. 175 (1909).

43. 312, U.S. 496 (1941).

44. Robert D. SCAPHIRO, op.cit., p. 1.419.

45. Así lo recomienda el Tribunal Supremo con preferencia sobre la abstención para estos casos. Cfr. Arizonans for Oficial English v. Arizona, 520 U.S. 43, 76 (1997). 
- El Supplemental Jurisdiction Statute de $1990^{46}$, permitía a un tribunal inhibirse del conocimiento de un recurso que "conllevara cuestiones nuevas o complejas relativas al Derecho del Estadon. A través de este método de declinación de jurisdicción suplementaria, muchos tribunales cumplen con la filosofía de la sentencia Siler de evitar cuestiones constitucionales federales innecesarias. El problema es que la jurisdicción suplementaria se creó para permitir el acceso a la jurisdicción federal sin necesidad de separar los recursos en cuestiones estatales y federales. Así se superaba el problema de que cuando un juez federal se abstenía, prácticamente cerraba la puerta a los litigantes para reclamar la protección de derechos reconocidos por la Constitución federal ante la jurisdicción federal. En el momento en que un juez declina la jurisdicción suplementaria, prácticamente los efectos son idénticos a los de la abstención.

- La declinación de jurisdicción suplementaria significa una elección arbitraria del juez para evitar una sentencia federal. Frente a esa arbitrariedad, el Tribunal Supremo determinó en Pennhurst State School \& Hospital v. Holderman ${ }^{47}$, que la undécima enmienda permitía un enjuiciamiento federal de los recursos basados en el derecho constitucional estatal interpuestos contra el propio Estado, según la interpretación de que el incumplimiento de la constitución estatal por una rama del Estado conlleva intereses atinentes a toda la Federación. En cambio, si el recurso se presenta contra una subdivisión administrativa del Estado, como por ejemplo un municipio, la undécima enmienda no contiene norma constitucional alguna al respecto.

- El Tribunal Supremo ha determinado que la abstención es apropiada cuando se trate de disposiciones constitucionales concretas que integran toda una regulación (regulatory regime) sin que existan disposiciones paralelas en la Constitución federal ${ }^{48}$.

Por tanto, la jurisdicción federal, cuando se invoquen frente a ella cuestiones que incumban a ambos órdenes jurisdiccionales, federal y estatal, puede hacer tres cosas:

- Decidir el recurso relativo a la constitución federal, bien:

a) rehusando abstenerse; bien

b) declinando ejercer la jurisdicción suplementaria en la cuestión relativa al Derecho estatal (sentencia Pennburst).

- Abstenerse para permitir al tribunal estatal decidir la cuestión constitucional (sentencia Pullman).

- Resolver ella misma la cuestión basada en el Derecho estatal (sentencia Siler).

En relación con el segundo de los aspectos, existe una notable discrepancia entre la elaboración doctrinal y la posición del Tribunal Rehnquist. Efectivamente,

46. 28 U.S.C., parágrafo 1.367.

47. 465 U.S. 89 (1984).

48. Reetz v. Bozanich, 397 U.S. 82 (1970). 
Frank B. Cross entiende que el principio federal de la Constitución americana, no ha tenido ni puede tener un significado legal tendente a limitar el poder del Gobierno central $^{49}$. Que el principio federal ha tenido un uso más político que jurídico en la historia de los Estados Unidos parece claro; fue el principio invocado por los Estados sureños para mantener la esclavitud primero, y la segregación después; igualmente, fue invocado en la batalla para frustrar las reformas del New Dea $\bar{b}^{\circ}$. Posteriormente, cuando el Tribunal Supremo se fue llenando de jueces conservadores, el Juez Brenan y otros constitucionalistas liberales dieron a luz un New Federalism para la protección de los derechos individuales a través de las constituciones estatales, que debían ser interpretadas en el sentido de otorgar una mayor protección que la contenida en la Constitución federal, o en el sentido de proteger derechos no reconocidos por ésta ${ }^{51}$. Frente a ello, la sentencia del caso Michigan v. Long ${ }^{52}$, determinó que el Tribunal Supremo presumiría que una decisión de un tribunal estatal tutelando derechos fundamentales, se basa en la Constitución federal, $y$, por tanto, que puede ser modificada por el propio Tribunal Supremo, a no ser que en la motivación el tribunal estatal demuestre claramente (siempre a juicio del Tribunal Supremo) que la decisión se deriva de la constitución estatal. Lo que, como es obvio, debilita notablemente las fronteras entre jurisdicciones.

Otro aspecto a tener en cuenta en la capacidad o incapacidad del principio federal para limitar el poder del gobierno central, es la posición institucional del Tribunal Supremo y de los tribunales federales, su ubicación orgánica. Y ello porque son los jueces federales quienes, en un sistema de common law, deben crear la base sobre la que encontrarán aplicación los límites derivados del principio federal. Sin embargo, pretender una vigorosa defensa judicial de dicho principio es ingenuo, ya que, a este respecto, los tribunales federales son agencias del gobierno federal ${ }^{53}$. Y ello por varias razones:

- En primer lugar, si bien, como hemos visto, han existido intentos de limitar el poder judicial a través del principio federal, este principio "has not been used by the judiciary as a limit on the federal legislative power ${ }^{54}$. Ello se explica porque

49. Frank B. Cross, Realism About Federalism, 74 New York Law Review, 1999, p. 1.304. Según este autor, el proceso de decisión judicial es esencialmente ideológico, con lo que una doctrina sobre el federalismo no es en sí misma fuerte o débil, sino que la aplicación de dicha doctrina dependerá de la composición ideológica del tribunal y de la implicación ideológica de los casos que en ella se presenten (p. 1.313). Sobre la concepción que del proceso judicial tiene este autor, cfr. Marcos CRIAlDO y Antonio DE CABO, "Actualidad constitucional en Estados Unidos en 1998", Teoría y Realidad Constitucional, cit., pp. 297 y ss.

50. Cfr. Erwin CHEMERINSKy, The Values of Federalism, 47 Florida Law Review, pp. 499 ss, donde afirma que el federalismo históricamente "has been used as political argument primarily in support of conservative causes" (p. 499).

51. Cfr. William J. BRENAN, State Constitutions and the Protection of Individual Rights, 90 Harvard Law Review, 1977, pp. 489 y ss. Desde un punto de vista histórico y valorativo de la efectividad de tal doctrina, cfr. Ellen D. PETERS, Capacity and Respect: A Perspective on the Historic Role of the State Courts in the Federal System, 73 New York Law Review, 1998, pp. 1.065 y ss.

52. 463 U.S. 1032 (1983).

53. Frank B. Cross, op.cit., p. 1.314.

54. Erwin Chemerinsky, op.cit., p. 505. 
desde la creación de la federación estadounidense se consideró que los intereses de los Estados estaban ampliamente protegidos por el desarrollo del proceso político en el nivel federal de gobierno, y no requerían protección judicial ${ }^{55}$.

- La dependencia de los jueces del Presidente y del Congreso se concreta en tres órdenes de factores:

1) El sistema de designación. Cuando los jueces son promovidos a los tribunales de apelación, el Congreso cuenta con una batería de casos relativos a la aplicación del principio federal sobre los que juzgar. Si las decisiones de los jueces en estos casos son contrarias a los intereses hegemónicos representados en el poder legislativo, difícilmente serán promovidos al Tribunal Supremo o a otros puestos de designación política.

2) El congreso puede controlar el poder judicial disminuyendo legalmente la jurisdicción de los tribunales federales ${ }^{56}$.

3) Competencia presupuestaria. De la competencia presupuestaria del Congreso dependen los fondos de los tribunales, así como los sueldos y complementos de los jueces ${ }^{57}$. El Congreso no puede reducir el sueldo de los jueces (art. III de la Constitución), pero sí puede congelarlo (Power of the Purse). Además de prohibir directamente la concesión de fondos para proyectos particulares promovidos o favorecidos por jueces, igualmente puede manifestar su general descontento con la marcha del poder judicial federal mediante una reducción general de las asignaciones de gasto (appropriations power) ${ }^{58}$.

- La décima enmienda de la Constitución determina: "The powers not delegated to the United States by the Constitution, nor prohibited by it to the States, are reserved to the States respectively, or to the peoplem. Si bien debería haber sido la base no sólo del reparto competencial, sino también de la protección de las competencias otorgadas a los Estados, casi nunca ha sido utilizada para restringir el poder nacional ${ }^{59}$.

55. Cfr. Garcia v. San Antonio Metro, Transit. Auth., 469 U.S. 528, 552 (1985) donde se declara que los intereses de los Estados "are more properly protected by procedural safeguards inherent in the structure of federal system than by judicially created limitations on federal power".

56. Cfr. Vicki C. Jackson, Congressional Control of Jurisdiction and the Future of the Federal Courts-Opposition, Agreement and Hierarchy, 86 Georgia Law Review, 1998, pp. 2.445-2.448, donde señala cómo recientemente se ha ido incrementando la utilización de este poder del Congreso.

57. Frank B. CROSs, op.cit., p. 1.317.

58. Cfr. Kate SMITH, Congress' Power of the Purse, 97 Yale Law Journal, 1988, pp. 1.343 y ss y Todd A. Peterson, Controlling the Federal Court Through the Appropriations Process, 1998 Wisconsin Law Review, pp: 993 y ss. Esta dependencia hace que los jueces tengan que medir muy bien los intereses que defender mediante un eventual enfrentamiento con el resto de los poderes del Estado, y entre estos intereses puede que a menudo no se encuentre la defensa de los Estados, que les haría malgastar gran parte del peso político necesario para la defensa de intereses más cercanos a la clase judicial.

59. Frank B. CROSs, op.cit., p. 1.324, donde prueba cómo nunca ha sido utilizada para oponerse a la extensión del poder del Congreso a través de la ampliación de la Commerce Clause. El juez Powell, en su voto particular a la sentencia Garcia v. San Antonio Metro [Transit. Auth., 469 U.S. 528 , 560 (1985)], declaró que el Tribunal había convertido la enmienda en una "retórica sin sentido". 
Los intentos doctrinales de promover un control jurisdiccional de la acción legislativa del Congreso también plantean otro tipo de problemas. La propuesta de Jackson de una revisión judicial del proceso legislativo del Congreso cuando se refiera a competencias sobre las que, según el principio federal, podrían legislar los Estados, incide enormemente sobre el principio de separación de poderes. En sustancia, el Congreso debería justificar razonada y razonablemente que la acción federal es justa y necesaria ${ }^{60}$, términos que, sometidos a control jurisdiccional, acabarian cobrando el sentido que los jueces quisieran darle y, por tanto, acabarían generando un due process al control de racionalidad, es decir, un procedural review of congressional action ${ }^{61}$.

Desde el punto de vista jurisprudencial, sin embargo, el segundo de los aspectos citados (el papel del Congreso en la definición de las libertades y su posible control por parte del Tribunal Supremo atendiendo al principio federal) ha alcanzado actualidad como consecuencia de la aprobación (y posterior anulación) en 1993 de la Religious Freedom Restoration Act (RFRA) ${ }^{62}$ en el sentido de una vigorosa aplicación del principio federal como limitación del poder del Congreso. Esta norma aprobada casi por unanimidad (unanimidad en la Cámara de Representantes, 97 a 3 en el Senado) pretendía defender la libertad religiosa frente a posibles violaciones por parte de los estados. Se daba así cumplimiento a la sección 5 de la Enmienda 14 ("The Congress shall have power to enforce, by appropiate legislation, the provisions of this article"). Es decir, el Congreso se ocupaba de definir el ámbito del derecho y de poner el remedio frente a sus violaciones en lugar de dejar tal función (exclusivamente) al Tribunal Supremo. Éste reaccionó en City of Boerne v. Flores [521 U.S. 507 (1997)] anulando esta norma alegando que los poderes del Congreso eran exclusivamente "remedial", es decir, "limitados a imponer una legislación que directa o indirectamente aplicase derechos judicialmente definidos frente a la interferencia estatal ${ }^{63}$. Lo que, en definitiva, se discute es el sentido de la doctrina sentada por el Juez Marshall en McCulloch v. Maryland ${ }^{64}$ según la cual el Congreso podrá desarrollar sus competencias constitucionales mediante una legislación "apropiada". Es decir, la conveniencia de aplicar una revisión judicial deferente según el principio de que las leyes del Congreso "Let the end be legitimate, let it be within the scope of the constitution, and all means which are appropiate, which are plainly adapted to that end, which are not prohibited, but consist with the letter and spirit of the constitution, are constitucional". En este caso, se trata de contrastar la defensa de los derechos de la decimocuarta enmienda con el principio federal. Tanto la ley ${ }^{65}$

60. Vicki C. JACKSON, Federalism and the Uses and Limits of Law, op.cit., p. 2.240.

61. Que es lo que venía a instaurar el Tribunal Supremo en la denostada Lochner v. New York, 198 U.S. 45 (1905).

62. 42 USC $\$ 2000 \mathrm{bb}(1994)$.

63. Steven A. ENGEL, The McCulloch Theory of the Fourteenth Amendment: City of Boerne v. Flores and teb Original Understanding of Section 5, The Yale Law Journal, vol. 109, p. 116 y n. 11.

64. U.S. (4 Wheat.) 316 (1819).

65. Erwin CHEMERINSKY, The Religious Freedom Restoration Act is a Constitutional Expansion of Rights, 39 WM. \& Mary L. Rev. 601, 610-14 (1988); Robert Hoff, Losing our Religion: The Constitutionality of the Religious Freedom Restoration Act Pursuant to Section 5 of the Fourteenth Amendment, 64 
como la sentencia ${ }^{66}$ han dado lugar a un importante debate doctrinal que, por el momento culmina en el trabajo de A. Engel que considera 1) que Boerne supone una violación de la doctrina McCulloch; 2) que la enmienda 14 encomienda la defensa de los derechos civiles tanto al poder judicial como al Congreso y 3) que la desconfianza frente a la creación jurisprudencial de derechos sin base textual constitucional pierde casi toda su razón de ser cuando es el legislador nacional el que los crea. La Enmienda Decimocuarta otorgó al Congreso la capacidad debatir y alcanzar el consenso sobre las libertades civiles merecedoras de protección, ${ }^{67}$.

El Tribunal Supremo, sin embargo, ha insistido en su línea federalista incluso más allá de la doctrina Boerne. Efectivamente, dando cumplimiento a la previsión del Juez Stevens en su voto particular a Seminole Tribe v. Florida [517 U.S. 44 (1996) at 77 (Stevens, J., dissenting)], de que la decisión de impedir el uso de los poderes del artículo I para excluir la inmunidad de la Undécima Enmienda impediría al Congreso establecer un foro federal" para las acciones contra los estados en un elevado número de materias [tales como quiebra, medio ambiente, copyright y patentes, Florida Prepaid Postsecondary Education Expense Board v. College Savings Bank (119 S. Ct. 2199 (1999)], anuló la Patent and Plant Variety Protection Remedy Clarification Act ("Patent Remedy Acts) ${ }^{68}$ en una controvertida decisión dividida. Conviene detenerse en esta sentencia porque avanza una jurisprudencia que, de consolidarse ${ }^{69}$, podría estar anunciando una línea que, en definitiva, busca una situación de desregulación característica del último capitalismo posmoderno. Desde 1987 el College Savings Bank (CSB) ofrecía unos seguros para pago de la educación universitaria que patentó. En 1988, una agencia estatal [la Florida Prepaid Postsecondary Education Expense Board ("Florida Prepaid»)] comenzó a ofrecer el mismo producto. El CSB demandó a Florida Prepaid por violación de patente en 1994, dos años después de la aprobación de la Patent Remedy Act que establecía que los estados y sus agencias carecían de inmunidad soberana frente a violaciones de patentes ante los tribunales federales. Como consecuencia de Seminole Tribe, Florida Prepaid solicitó el archivo de la causa alegando que el Congreso carecía de facultades para dictar la citada Ley. El Tribunal del Distrito rechazó

Brook. L. Rev. 377 (1998); Matt PAWA, When the Suprem Court Restricts Constitutional Rights, Can Congress Save Us? An Examination of Section Five of the Fourteenth Amendment, 141 U. Pa. L. Rev. 1029 (1993); Jay S. BYBEE, Taking Liberties with the First Amendment: Congress, Section 5, and the Religious Freedom Restoration Act, 48 Vand. L. Rev., 1539 (1995); Bonnie I. ROBIN-VERGEER, Disposing of the Red Herrings: A Defense of the Religious Freedom Restoration Act, 69 S. Cal. L. Rev. 589 (1996).

66. Ira C. Lupu, Why the Congress Was Wrong and the Court Was Right: Reflections on City of Boerne v. Flores, 39 WM. \& Mary L. Rev. 793, 811-12; Michael W. McConnELL, Institutions and Interpretation: A Critique of City of Boerne v. Flores, 111 Harv. L. Rev. 153, 185-188 (1997); Rachel ToKER, Tying the Hands of Congress: City of Boerne v. Flores, 33 Harv. C.R.-C.L. L. Rev. (1988); Akhil ReED AmAr, Intratextualism, 112 Harv. L. Rev. 747 y ss (1999); William G. Buss, An Essay on Federalism, Separation of Powers, and the Demise of the Religious Freedom Restoration Act, 83 Iowa L. Rev. (1998).

67. Steven A. ENGEL, cit., p. 154.

68. Pub. L. No. 102-560 Stat. 4230 (1992).

69. Lo que parece estar sucediendo, cf. Alden v. Maine [119 S. Ct. 2240 (1999)], en la que se establece que la inmunidad soberana de los Estados impide que el Congreso, actuando con los poderes del artículo I, someta a un Estado que no consiente a litigios privados por daños antes sus propios tribunales. 
la petición aplicando el test de Seminole Tribe. A saber, voluntad inequívoca de suprimir la inmunidad más actuación en ejercicio válido de sus poderes. Las patentes han sido siempre consideradas propiedad, y la protección frente a su privación caería, por tanto, dentro del ámbito de la Enmienda 14 y, consiguientemente, de su sección 5. El Federal Circuit confirmó la decisión en decisión unánime. La Corte de Apelación volvió a confirmar y, confiando en Boerne, estableció que los medios escogidos por el Congreso eran congruentes con el mal causado (privación estatal de la patente sin debido proceso). Sin embargo, el Tribunal Supremo en una decisión 5-4 casó todas estas decisiones y estableció que el Congreso carecía de poderes según la Sección 5 para suprimir la inmunidad soberana frente a litigios por violación de patentes. La razón principal del Tribunal fue que "la Patent Remedy Act no responde a una historia de privación extendida y persistente de derechos constitucionales" necesaria para "la legislación profiláctica de la Sección 5". Según el Tribunal, de los antecedentes no se derivaba la conclusión del Congreso de que los estados estuvieran privando a los dueños de patentes de su propiedad sin debido proceso amparándose en la inmunidad soberana en las acciones ante tribunales federales. Además, no existiría propocionalidad puesto que en lugar de limitar el alcance de la ley a las violaciones intericionales, se extendía a todas ellas. La Patent Remedy Act estaría "tan fuera de proporción frente a un supuesto objetivo preventivo o de resarcimiento que no puede entenderse como respuesta a, o diseñada para prevenir, un comportamiento inconstitucional.. Lo que importa destacar, como hizo el Juez Stevens en su voto particular, es que los casos Boerne y actual son radicalmente distintos, y éste supone un paso mucho más allá de aquél. Boerne anula la RFRA surgida como respuesta directa a Employment Division v. Smith [494 U.S. 872 (1990)] que establecía que los estados no tenían que demostrar un "compelling interest" para justificar leyes de aplicación general que incidentalmente supusieran una carga al libre ejercicio de la religión. La RFRA establecía justamente este requisito cuando se impusiera una carga sustancial en el ejercicio de la religión a alguna personaw. Estaban, por tanto, aquí en juego delicados equilibrios de federalismo, separación de poderes y libre ejercicio de la religión. Lo que el Tribunal trató de decidir en Boerne (no sin oposición, como hemos visto) es que el Congreso no podía establecer legislación sustantiva con base en la Sección 5, es decir no podía decidir "what the law is" (tarea que corresponde a los Tribunales) sino sólo poner los medios frente a su violación. Igualmente, el Tribunal trató de evitar que el Congreso interfiriera con las prerrogativas tradicionales de los Estados y su autoridad general para regular la salud y bienestar de sus ciudadanos". El caso con la Patent Remedy Act es totalmente diferente ya que ni expande derechos constitucionales (frente a los tribunales) ni impone obligaciones adicionales a los estados. En primer lugar, el artículo I concede expresamente al Congreso el mandato de legislar en materia de patentes y, dado que los tribunales ${ }^{70}$ han reconocido que las patentes son propiedad, el Congreso no interfirió con el poder judicial definiendo derechos sustantivos a efectos 
de la Enmienda 14. Igualmente, en lo que se refiere al federalismo, dado que el Congreso puede legislar en patentes según el artículo I, los estados están obligados a cumplir dichas normas: La Enmienda 11 puede impedir los litigios en los tribunales federales pero no autoriza a violar la ley federal, de forma tal que la Ley no impone obligaciones nuevas, se limita a impedir o remediar por vía de la Sección 5 el no cumplimiento (enforcement) de las ya existentes.

En resumen, Seminole Tribe, Boerne, Florida Prepaid y Alden se han convertido en un complejo conjunto de decisiones que además de representar el tema por excelencia del Tribunal Rehnquist ${ }^{11}$ ha hecho inútil los intentos de Congreso de buscar compensación para los individuos ante los daños sufridos en una gran variedad de programas federales, sometiendo a los Estados a jurisdicción bien ante los tribunales federales, bien ante los propios tribunales estatales. Lo paradójico de esta situación es que pone en entredicho la propia lógica del Tribunal. Dado que, reiteradamente, se afirma que la defensa del federalismo tiene por objeto la división del poder y la promoción de la libertad ${ }^{72}$ no deja de ser sorprendente que su aplicación implique una situación en la que frente a violaciones de derechos concedidos, nadie (ni Federación ni Estado) responde.

\section{EDUCACIÓN, RACISMO Y FINANCIACIÓN}

La educación pública en Estados Unidos, con su permanente subfinanciación y su frecuente estigmatización social ha sido objeto de atención por parte tanto de la doctrina como de la jurisprudencia de forma recurrente. Ahora bien, en realidad, en ambos campos existían dos discursos diferenciados: el relativo a la necesaria financiación (pobre/rico) y el que se refiere a la segregación (blanco/negro). Una importante sentencia del Tribunal Supremo de Connecticut (Sheff $v$. O'Neill) ${ }^{73}$ ha impulsado en la doctrina norteamericana una importante línea de investigación que trata de unir ambos criterios y de estudiar las interrelaciones existentes entre ellos. Mary Jane Lee $^{74}$ ha estudiado las relaciones entre la citada sentencia y los esfuerzos desegregacionistas que iniciara el Tribunal Supremo en Brown v. Board of Education ${ }^{75}$ cuando afirmó que la existencia de instalaciones educativas para blancos y negros resultaba inherentemente desigual. Como es sabido, sin embargo, las diferencias entre escuelas a las que asisten blancos y negros persiste de forma alarmante. Y ello,

71. Cf. Marcia CoYle \& Harvey BERKMAN, Justices Weigh in on Side of States, N.Y. L.J., July 5, 1999 , A1.

72. Printz, 521 U.S. 898, 921, 922 (1997): la soberanía dual es suna de las protecciones estructurales de la Constitución a la libertad" que "ofrece una doble seguridad a los derechos del pueblo"; New York, 505 U.S. at 181: "La Constitución no protege la soberanía de los Estados en beneficio de los Estados o de los gobiernos de los Estados como entidades políticas abstractas, ni siquiera en beneficio de los funcionarios públicos que los gobiernan. Al contrario, la Constitucion divide la autoridad entre gobiernos federal y estatal para proteger a los individuos".

73. 678 A.2d 1267 (Conn. 1996).

74. How Sheff revives Brown: reconsidering desegregation's role in creating equal educational opportunity, New York Law Review, vol. 74:485-528.

75. 347 U.S. 483 (1954). 
de manera especialmente intensa en las escuelas de los distritos centrales de las ciudades (a las que sólo asiste un 3,3 por ciento de alumnos blancos ${ }^{76}$ ). Efectivamente, en éstas se concentran los peores resultados educativos. La importancia de Sheff estriba en haber reconocido como parte del derecho a iguales oportunidades educativas, no sólo las discriminaciones de iure, sino también las de facto. Es decir, el hecho de que tales escuelas resulten, de hecho, escuelas para minorías en las que (por ello mismo) se concentran especiales niveles de pobreza y desintegración social (en 1994, el 46 por ciento de los niños negros vivían en la pobreza, el triple que sus homólogos blancos). Por otra parte, el hecho de que Sheff remita la solución al legislador permite augurar una mayor aceptación social de las posibles medidas correctoras que las decisiones obligatorias (el denostado "busing") emitidas por los tribunales. Desgraciadamente, la respuesta legislativa no ha seguido las indicaciones de la Sentencia, ya que en lugar de atacar el núcleo del problema (la segregación racial), ha tratado de incrementar las posibilidades de que se produzcan traslados interdistritales entre los estudiantes y de paliar las disparidades entre distritos urbanos y suburbanos.

En esta misma área, sin duda, el esfuerzo académico de mayor calado es la serie de artículos del profesor James $\mathrm{E}$. Ryan en los que ha tratado de ofrecer tanto un balance histórico de la lucha por la iguladad de oportunidades educativas como una propuesta política viable para el presente. Se trata de Sheff, Segregation, and School Finance Litigation ${ }^{77}$, School, Race, and Money ${ }^{78}$ y The Influence of Race in School Finance Reform ${ }^{79}$. En el primero de ellos, James E. Ryan trata de explicar y justificar la decisión de Sheff en un caso (el de Hartford) en el que las escuelas en cuestión (de mayoría negra) recibían una mayor subvención que la media y que, sin embargo, representan uno de los peores ejemplos de rendimiento académico. Fue esta constatación la que llevó tanto al Tribunal como a él mismo, a considerar medidas que ataquen directamente a la segregación. $\mathrm{O}$, dicho de otro modo, una educación segregada de facto no puede ser una educación de calidad lo que pemite adoptar este tipo de medidas, incluso si las constituciones estatales no establecen nada sobre la segregación (hay que recordar que sólo tres constituciones estatales incluyen este tipo de declaraciones), tendiendo un vínculo entre calidad o suficiencia educativas y desegregación. En el segundo de sus trabajos, James E. Ryan estudia el camino separado que han seguido los casos de "School Desegregation" frente a los de "School Finance». De este estudio histórico se extrae la consecuencia de que ante las dificultades que acarreaba una desegregación impuesta judicialmente el énfasis se fue trasladando hacia la existencia de financiación suficiente incluso en presencia de escuelas segregadas. La evidencia ha demostrado que estas "Ghetto Schools" no ofrecen buenos resultados académicos por razones semejantes a las expuestas en el trabajo de Mary Jane Lee citado. Ello abre la posibilidad, basándose en Sheff de abrir

76. Kevin Brown, After the Desegregation Era: The Legal Dilemma Posed by Race and Education, 37 St. Louis U.L.J., 898, nota 5.

77. New York Law Review, vol. 74, pp. 529-573 (1999).

78. The Yale Law Journal, vol. 109, pp. 249-316 (1999).

79. 98 Mich. L. Rev., nov. 1999. 
una Fourth Wave of School Finance Litigation que se refiera no tanto a cuánto se gasta en cada escuela sino a la existencia o no de escuelas de minorías raciales. En el tercero de sus trabajos, se insiste nuevamente en las relaciones entre los problemas raciales y los de financiación demostrando que los distritos escolares de minorías raciales, en especial los de áreas urbanas, no obtienen buenos resultados en sus reclamaciones judiciales sobre financiación, y que las cuestiones raciales parecen desempeñar un papel fundamental en las decisiones judiciales y en las reacciones legislativas a estas mismas decisiones.

\section{JURISPRUDENCIA ${ }^{80}$}

EN RELACIÓN CON LA LIBERTAD DE EXPRESIÓN

Buckley v. American Constitutional Law Foundation, 119 S. Ct. 636 (1999)

En 1994, la American Constitutional Law Foundation (ACLF) impugnó la normativa sobre iniciativa legislativa popular (ballot initiative) de Colorado alegando la inconstitucionalidad de algunos de sus requisitos que, en su opinión, restringían la libertad de expresión. A saber: la mayoría de edad para los encargados de la iniciativa (circulators), la exigencia de que sean votantes censados, la restricción a seis meses del período de recogida de firmas, la obligación de llevar indicadores con sus nombres, la obligación de anexar a cada petición una declaración con sus nombres, direcciones y la declaración de haber leído y entendido las normas sobre iniciativas; la obligación para los promotores de revelar las cantidades pagadas para la recogida de firmas, y los nombres y datos personales de los encargados de hacerlo, información que debía repetirse mensualmente durante el período de recogida de firmas. El tribunal del distrito anuló la exigencia de distintivos y parte de la obligación de revelación de datos pero admitió la de edad, declaración y el período de recogida. El Tribunal Estadounidense de Apelaciones para el Décimo Circuito confirmó en parte y rechazó en parte. Admitió la edad, tiempo de recogida y la declaración pero anuló la exigencia de registro en el censo y en parte lo relativo a los distintivoś y las obligaciones de revelación de información. El Tribunal Supremo confirmó esta decisión.

\section{EN RELACIÓN CON EL DUE PROCESS}

City of Chicago v. Morales, 119 S. Ct. 1849 (1999)

El 17 de junio de 1992, el Ayuntamiento de Chicago dictó una ordenanza «antigang loitering" (bandas merodeadoras). En ella se definía merodear ("loitering")

80. Como en anteriores revisiones, el análisis de la jurisprudencia se realiza, básicamente, a partir de las reseñas de la Harvard Law Review. 
como "permanecer en un lugar sin propósito aparente" y se establecía que "cuando un oficial de policía observe a alguna persona de la que crea razonablemente se trata de un miembro de una banda criminal callejera merodeando en un lugar público con una o más personas, les ordenará que se dispersen y se retiren de dicha área. Quienes no obedezcan con prontitud esta orden, violarán el presente artículo" ${ }^{81}$. Durante los tres años de su aplicación se produjeron 89.000 órdenes de dispersión y más de 42.000 arrestos. El Tribunal de apelaciones del Estado declaró inconstitucional la ordenanza. El Tribunal Supremo de Illinois ${ }^{82}$ sostuvo dicha inconstitucionalidad, confirmada después por el Tribunal Supremo. El Tribunal Supremo desarrolló la doctrina de Conally v. General Constr. Co. ${ }^{83}$ según la cual una norma penal "tan vaga que personas de inteligencia normal se vean obligadas a preguntarse por su significado y a diferir en cuanto a su aplicación" supone una violación del debido proceso, estableciendo un doble test para el concepto de vaguedad. Conforme al mismo, la vaguedad provocaría inconstitucionalidad cuando "En primer lugar, no contenga una afirmación tal que permita a gente corriente comprender qué conducta prohíbe; segundo, pueda autorizar o incluso estimular una aplicación arbitraria o discriminatoria". Aunque en decisión dividida, el Tribunal Supremo sostuvo que la definición de "merodear" de la ordenanza no superaba dicho tèst.

\section{DETENCIÓN Y REGISTRO}

\section{Wyoming v. Houghton, 119 S. Ct. 1297 (1999)}

El 23 de junio de 1995, una patrulla de policía detuvo a un automóvil que circulaba con exceso de velocidad. Los agentes observaron una jeringuilla en el bolsillo del conductor. Preguntado sobre ella, respondió que la utilizaba para consumir drogas. Los agentes ordenaron a los ocupantes (el conductor, su novia y una tercera ciudadana) que abandonaran el coche y procedieron a su registro. En el asiento posterior encontraron un bolso de mujer. Al abrirlo, hallaron la documentación de esta tercera persona y posteriormente una cartera con metanfetamina. Los agentes detuvieron al tercer ocupante del coche y se le procesó por tenencia de sustancias estupefacientes. Se discute la extensión de la llamada Automobile exception (reconocida por primera vez en Carroll v. United States, 267 U.S. 132, 153 (1925)) a la necesidad de orden de registro de la Cuarta Enmienda. La procesada solicitó la anulación del cuerpo del delito alegando que en el momento del registro, el único implicado en posible actividad criminal era el conductor y que, dado que el bolso claramente no le pertenecía (era de mujer y en su interior se halló, en primer lugar, la documentación de la ahora procesada), éste carecía de "probable cause". El tribunal en la instancia basándose en California v. Acevedo, 500 U.S. 565 (1991) (que

81. Chicago, Ill., Mun. Code $₫ 8-4-015$ (1992).

82. 687 N.E.2d 53 (Ill. 1997).

83. 269 U.S. 385, 391 (1925). 
establece que si existe "probable cause" de que un contenedor de un coche contenga contrabando puede registrarse sin orden, aunque no haya "probable cause" para registrar todo el coche) decretó que dado que había causa para registrar el coche también existía para registrar cualquier contenedor que pudiera ocultar lo buscado. El Tribunal Supremo de Wyoming casó la decisión basándose en United Sates $v$. Ross 456 U.S. 798 (1982) ("el alcance de un registro sin orden en un automóvil... viene determinado... por los lugares en los que exista probable cause para creer que [dicho objeto] pueda hallarse") y Acevedo, afirmando que el alcance de los registros sin orden es el que tendría de haberse emitido tal orden. Dado que según Ybarra v. Illinois, 444 U.S. 85 (1979) y United States v. Di Re, 332 U.S. 581 (1948) las órdenes de registro no se extienden a los acompañantes o invitados del registrado (el registro del bar no se extiende a sus clientes; el registro del conductor de un coche no se extiende al registro personal de los pasajeros), el tribunal sostuvo que la "probable cause" de que el conductor del coche fuera culpable de un delito no autorizaba a registrar las propiedades de los pasajeros, una vez que los agentes tenían noticia de que dichas propiedades estaban "en posesión o bajo el control" de los mismos. El Tribunal Supremo anuló la decisión del tribunal del estado. Según el Alto Tribunal para determinar si un determinado registro viola la Cuarta Enmienda debe realizarse un doble test. En primer lugar, determinar si el registro se habría considerado legal en el momento en que se estableció la Enmienda. Si dicha investigación no ofrece respuesta, el tribunal debe realizar un «balancing tesț" (ponderación) para determinar si la "promoción de un interés estatal legítimo" justifica la "intrusión en la privacidad individual". Según el Tribunal, los pasajeros de un automóvil tienen una "reduced expectancy of privacy" en relación con las propiedades que transportan en los coches, derivada del hecho de que los automóviles no suelen utilizare como depósito para los efectos personales, la amplitud de los controles estatales asociados al tráfico y el riesgo de que un accidente deje el contenido del coche abierto a la inspección pública. Según el Tribunal hay que distinguir entre registros personales (más intrusivos) y registros de propiedades individuales (menos intrusivos), teniendo en cuenta que la alegación de propiedad podría impedir la mayor parte de los registros en los coches (el conductor podría alegar que todo cuanto hay en el coche pertenece a terceros). El Juez Stevens en voto particular declaró que esta sentencia suponía abandonar el precedente que distinguía entre conductor y pasajeros y sustituirlo por la distinción entre registros personales y de propiedades, al tiempo que afirmaba que el registro de un abolso o de una cartera supone una intrusión en la privacidad que puede ser tan importante" como la de un registro personal.

\section{Minnesota v. Carter, 119 S. Ct. 469 (1998)}

En Minnesota v. Olson (495 U.S. 91 (1990)), el Tribunal Supremo había aclarado que los "huéspedes que pasan la noche" poseen una "reasonable expectation of privacy" en dicho domicilio y que, por tanto, quedan cubiertos por la Cuarta Enmienda (inviolabilidad de domicilio). En el presente caso se trata de aclarar la 
situación de los invitados durante un período menor. El 15 de mayo de 1994, un informante comunicó a la policía que en cierta vivienda se estaba procediendo al "embolsado" de un polvo blanco. El agente de policía se acercó a la ventana y, aunque las persianas estaban bajadas, observó a través de una agujero tal actividad. Lo comunicó a comisaría y se obtuvieron las órdenes de registro. Dos de los ocupantes abandonaron la casa y fueron detenidos incautándoseles cocaína. El tercero, arrendatario de la vivienda, fue detenido en la casa con idéntico resultado. Los dos primeros "habían ido al apartamento con la única intención de empaquetar la cocaína" y "nunca habían estado antes en el apartamento, en el que sólo habían permanecido dos horas y media". En la vista, se solicitó la anulación de las pruebas dado que el registro del coche y del apartamento se derivaba de un registro visual ilegal del apartamento a través del agujero en la persiana. El tribunal en la instancia negó tal anulación invocando que su condición de visitantes temporales les privaba de "reasonable expectation of privacy" y, por tanto, de la garantía de la Cuarta Enmienda. La Corte de Apelación de Minnesota confirmó la decisión. El Tribunal Supremo de Minnesota, en decisión dividida, la casó extrapolando el caso Olson a las visitas de corta duración. El Tribunal Supremo anuló la decisión del Supremo de Minnesota estableciendo que los imputados carecían de "reasonable expectation of privacy" por tres razones. Primera, no se trataba de huéspedes que pasan la noche, sino que sólo habían pasado en la casa dos horas y media. Segundo, carecían de "relación" o "conexión" con el arrendador, al margen del tiempo que habían pasado "embolsando" cocaína. En tercer lugar, los imputados utilizaron el apartamento exclusivamente para sus negocios (business transaction) por lo que su expectativa de privacidad era menor, ya que "la expectativa de privacidad en locales comerciales... es diferente e, indudablemente, menor que la que se da en un domicilio particular». En resumen, según la nueva lectura de la Cuarta Enmienda: los invitados durante períodos breves en casa de un virtual extraño con propósito de negocios carecen de una expectativa razonable de privacidad y, por tanto, no están cubiertos por la inviolabilidad de domicilio.

\section{EN RELACIÓN CON LA CONFRONTATION CLAUSE DE LA SEXTA ENMIENDA}

\section{Lilly v. Virginia, 119 S. Ct. 1887 (1999)}

Según la "Confrontation clause" el acusado tiene derecho "a confontar a los testigos de cargo". Con frecuencia se alega violación de esta cláusula cuando se $\mathrm{da}$ entrada en un procedimiento penal a declaraciones indirectas ("hearsay evidence" definidas como "declaraciones, distintas de las realizadas por el declarante al testificar en juicio, ofrecidas como evidencia para probar la verdad de la cuestión debatida"). El Tribunal Supremo ha establecido que ambas situaciones no son coextensas. En Obio v. Roberts (448 U.S. 56 (1980)) declaró que la aceptación de declaraciones indirectas no viola la confrontation clause cuando se trate de una excepción "firmemente establecida" o tenga "especiales garantías de fiabilidad". En la presente sentencia, el Tribunal estableció que la admisión de la confesión fuera 
de juicio de un cómplice detenido no constituye una excepción sfirmemente establecida". En diciembre de 1995, los hermanos Lilly y G.W. Barker cometieron durante dos días, en estado de intoxicación etílica, una serie de delitos incluyendo el secuestro y asesinato de un ciudadano. En el interrogatorio policial, dos de los imputados acusaron al tercero (uno de los hermanos Lilly) de ser el responsable del homicidio. En juicio, el principal testigo fue Barker, si bien la acusación reclamó el testimonio del segundo de los hermanos Lilly. Éste se negó a declarar contra sí mismo (Quinta Enmienda) y la Fiscalía presentó como prueba su declaración policial, como declaración de testigo no disponible contraria a su propio interés penal (una de las excepciones a las declaraciones indirectas). El principal imputado se opuso alegando que dicha declaración no era contraria al interés penal del declarante puesto que trasladaba la principal responsabilidad penal a otra persona. El tribunal no admitió la oposición y lo condenó a muerte. El Tribunal Supremo de Virginia confirmó la decisión, afirmando que se trataba de declaración contra el interés penal del declarante. Según el Tribunal la decisión era confiable en la medida en que le implicaba en numerosos delitos, además de existir otras pruebas independientes. Según la ley de Virginia nos encontraríamos ante una excepción "firmemente arraigada". El Tribunal Supremo casó la decisión. En primer lugar, apreció que el tratarse o no de excepción "firmemente arraigada" era cuestión federal y no estatal con lo que desautorizó el razonamiento del Tribunal Supremo de Virginia. Según el Tribunal Supremo la categoría "declaración contra el interés penal" es demasiado amplia para enfrentarla a la Confrontation Clause. Una de sus subdivisiones es la de las declaraciones de la acusación tendentes a probar la culpabilidad del imputado, presunto cómplice del declarante. Según el Tribunal estas declaraciones son "inherentemente no fiables" si no son sometidas a careo en la medida en que existe una motivación obvia en desviar la culpa hacia los cómplices, por lo que no resultan, sin ambigüedad, contrarias al propio interés penal. Esto no excluye que puedan ser fiables en algún caso, pero corresponde a la acusación probar el segundo requisito establecido en Roberts, es decir, que existen garantías específicas de fiabilidad. Lo que no se dio en el caso en cuestión, ya que lo que se exige no es que exista evidencia externa que confirme lo declarado, sino que existan elementos intrínsecos a la declaración que la hagan fiable. 